BRONCHIECTASIS

\title{
Non-tuberculous mycobacteria in patients with bronchiectasis
}

\author{
M Wickremasinghe, L J Ozerovitch, G Davies, T Wodehouse, M V Chadwick, \\ S Abdallah, P Shah, R Wilson
}

See end of article for authors' affiliations

......................

Correspondence to: Dr R Wilson, Host Defence Unit, Royal Brompton Hospital, Sydney Street London SW3 6NP, UK: r.wilson@rbh.nthames. nhs.uk

Received 11 May 2005 Accepted

10 September 2005

Published Online First 14 October 2005
Background: Non-tuberculous mycobacteria (NTM) are ubiquitous environmental organisms. Patients with pre-existing lung damage are susceptible to NTM, but their prevalence in bronchiectasis is unknown. Distinguishing between lung colonisation and disease can be difficult.

Methods: A prospective study of 100 patients with bronchiectasis was undertaken to evaluate the prevalence of NTM in sputum, and a retrospective analysis of clinical, microbiological, lung function and radiology data of our clinic patients with NTM sputum isolates over 11 years was performed.

Results: The prevalence of NTM in this population of patients with bronchiectasis was $2 \%$. Patients in the retrospective study were divided into three groups: bronchiectasis + multiple NTM isolates $(n=25)$, bronchiectasis + single isolates $(n=23)$, and non-bronchiectasis + multiple isolates $(n=22)$. Mycobacterium avium complex (MAC) species predominated in patients with bronchiectasis compared with non-bronchiectasis lung disease $(72 \% \vee 9 \%, p<0.0001)$. Single isolates were also frequently MAC (45.5\%). Multiple isolates in bronchiectasis were more often smear positive on first sample than single isolates $(p<0.0001)$. NTM were identified on routine screening samples or because of suggestive radiology. No particular bronchiectasis aetiology was associated with an NTM. Pseudomonas aeruginosa and Staphylococcus aureus were frequently co-cultured. Six (25\%) of multiple NTM patients had cavities of which five were due to MAC. Half the patients with multiple isolates were treated, mostly due to progressive radiology.

Conclusions: NTM are uncommon in non-cystic fibrosis bronchiectasis. Routine screening identifies otherwise unsuspected patients. MAC is the most frequent NTM isolated.
$\mathrm{N}$ on-tuberculous mycobacteria (NTM) are ubiquitous environmental organisms that sometimes cause respiratory disease, usually in patients with preexisting damage. Patients with bronchiectasis, as with other chronic lung diseases, are predisposed to infection with NTM. ${ }^{2}$ The symptom profile of NTM pulmonary infectionsuch as increased sputum production, cough, breathlessness and haemoptysis-is similar to bronchiectasis, making differentiation between active infection and colonisation difficult. Furthermore, some NTM infections cause bronchiectasis, ${ }^{3}$ so a radiological diagnosis of NTM infection is difficult to make in the presence of underlying disease. Similarly, primary infections that have caused bronchiectasis may be impossible to differentiate from secondary infections of bronchiectasis due to another cause.

NTM are inhaled as aerosol droplets so may be cultured if a sputum sample is obtained soon after environmental exposure. This probably accounts for most of the single isolates found. ${ }^{4}$ However, because single isolates could reflect a low level of infection which may increase, careful follow up with repeated sampling is required. Multiple isolates, with clinical evidence of disease, should be obtained before starting prolonged treatments that are sometimes poorly tolerated..$^{5-7}$

Few studies have undertaken a detailed analysis of NTM in the context of bronchiectasis. The prevalence of NTM in bronchiectasis may be higher than anticipated because of the non-specific symptoms and because routine screening is not usually undertaken. In one study, three cases of NTM were detected over 6 years in 91 patients with bronchiectasis. ${ }^{8}$ In another, NTM were found in $6 \%$ of bronchiectatic patients. ${ }^{9}$ No mycobacteria were isolated in a study of 150 patients over
3 years, ${ }^{10}$ but in this study sputum was sent only if no response to standard treatment occurred.

We have prospectively investigated the prevalence of NTM in two groups of bronchiectasis patients: (1) newly referred patients to our unit in whom NTM were unsuspected and (2) patients being followed up in our unit who had a previous negative sputum examination for acid fast bacilli (AFB). At the time of this study it was not our practice to screen for AFB routinely. In a retrospective study over 11 years we identified patients with and without bronchiectasis with NTM isolates who were seen in our clinic. We also studied different NTM species in the retrospective study with respect to high resolution computed tomographic (HRCT) features, lung function, and co-existent sputum pathogens. Lastly, we investigated how many patients were judged to require treatment for NTM infection and why.

\section{METHODS}

\section{Prospective study population}

A prospective analysis was performed of the prevalence of NTM isolates in sputum from 50 consecutive newly referred patients with bronchiectasis admitted to our minimal dependency unit for investigation (which is our usual practice for new patients) and 50 follow up bronchiectasis patients recruited from outpatient clinics because their condition was stable and they had a previous negative

\footnotetext{
Abbreviations: $A F B$, acid fast bacilli; $F_{1} V_{1}$, forced expiratory flow in 1 second; MAC, Mycobacterium avium complex; $\mathrm{MEF}_{50}$, maximum expiratory flow with $50 \%$ of vital capacity remaining in lung; NTM, nontuberculous mycobacteria; RV, residual volume; TLCO, carbon monoxide transfer factor
} 
sputum examination for AFB. Patients referred because of known mycobacterial infection and those with a positive sweat test indicative of cystic fibrosis were excluded. Sputum was sent for smear and culture examination on three separate occasions $(0,6$, and 12 months $)$. At the time of this study it was not our practice to screen routinely for NTM except during the patient's initial investigations in our unit.

\section{Retrospective study population}

A retrospective analysis over 11 years from 1991 to 2001 of patients in the Host Defence Unit with a positive NTM sputum culture was performed using the Royal Brompton Hospital Department of Microbiology database. Case notes were reviewed and patients allocated to the following groups: (1) patients with bronchiectasis and a single NTM isolate; (2) patients with bronchiectasis and multiple NTM isolates; and (3) patients with non-bronchiectasis lung disease and multiple NTM isolates.

All patients had daily sputum production and a history of recurrent infective exacerbations. The diagnosis of bronchiectasis was made by HRCT scanning. ${ }^{2}$ Patients with bronchiectasis and multiple isolates were analysed for age, sex, aetiology of bronchiectasis, whether the NTM infection was primary or secondary, past medical history, drug history, sputum microbiology, HRCT appearance, lung function, and drug treatment.

\section{Microbiology}

Samples were processed for mycobacteria using the modified Petroff's 4\% sodium hydroxide method. ${ }^{11}$ The processed samples were then inoculated onto two Lowerstein-Jensen slopes (one with pyruvate and one with glycerol). AFB positive smear samples were also inoculated into Middlebrook $>\mathrm{H} 12$ Bactec vials. Positive cultures were identified by biochemical and temperature tests and, where appropriate, Accuprobe. ${ }^{12}$ The criteria for multiple isolates were two isolates that were cultured more than 1 week apart. ${ }^{5}$ Single isolates were a single positive NTM culture listed in the microbiology database. The presence of negative cultures before and after the single isolate was confirmed and the presence or absence of smear positivity was documented. Sputum was sent at the same time as AFB examination for routine bacteriological examination. Pathogens cultured within 1 week of the first or single NTM isolate were documented and described as a co-cultured pathogen. These were described as chronic isolates if grown on more than two successive occasions.

\section{Lung function}

Airflow obstruction, gas trapping, and gas transfer were measured since these are usually impaired in bronchiectasis. ${ }^{13}$ Forced expiratory flow in 1 second $\left(\mathrm{FEV}_{1}\right)$, maximum expiratory flow with $50 \%$ of vital capacity remaining in lung $\left(\mathrm{MEF}_{50}\right)$, residual volume (RV), and carbon monoxide transfer factor (TLCO) were measured. Lung function nearest the time of the first isolate was compared with the most recent set of lung function data recorded.

\section{Radiology}

All HRCT scans were performed at the Royal Brompton Hospital. The timing of the first HRCT scan with respect to the first NTM isolate was calculated. The predominant location of bronchiectasis, severity, presence or absence of nodules, mucus plugging, cavitation, consolidation, infiltrates, and dyshomogeneity (air trapping) was assessed. ${ }^{13}{ }^{14}$

\section{Statistical analysis}

Group comparisons were made using $\chi^{2}$ or Fisher's exact test as appropriate. Lung function data were analysed using paired $t$ tests. A p value of $<0.05$ was regarded as statistically significant.
RESULTS Prospective study of NTM in sputum from patients with
bronchiectasis

Of the 50 newly referred patients with bronchiectasis, one had multiple Mycobacterium avium complex (MAC) isolates. This was a 52 year old woman referred because of poor symptom control and chronic Staphylococcus aureus infection. Investigation showed idiopathic bronchiectasis and an HRCT scan with an unusual pattern of bronchiectasis involving the anterior segment of the right upper lobe and right middle lobe with marked dyshomogeneity suggesting small airways disease. The sputum had not been previously investigated for mycobacteria. Another patient had a single $M$ chelonae isolate that was never repeated.

Of the 50 patients with bronchiectasis who were being followed up, one had multiple MAC isolates. This 64 year old woman had a 10 year history of allergic bronchopulmonary aspergillosis and seropositive rheumatoid arthritis. During initial assessment she was smear and culture negative for mycobacteria and had widespread severe bronchiectasis with a left apical cavity and mycetoma. She was stable over the next 8 years, after which she was enrolled into the study and her sputum sent for this purpose was smear and culture positive for MAC on all three occasions.

The prevalence of NTM in this population of patients with bronchiectasis in our Host Defence Unit was therefore 2\% over 1 year. The two patients were regarded as being colonised as their condition was well controlled with general treatment. The first patient improved with broad spectrum antibiotics and physiotherapy; the second patient remained stable for several years but then became more symptomatic and a new CT scan showed several cavitatory nodules. Treatment was commenced at that time.

\section{Demographic data of patients in retrospective study}

Seventy one patients with NTM isolates were identified over 11 years: 23 with bronchiectasis and single NTM isolates (single NTMB group), 25 with bronchiectasis and multiple NTM isolates (multiple NTMB group), and 22 with multiple NTM isolates and non-bronchiectatic lung disease.

There was a female preponderance in both the single (9 men, 14 women) and multiple NTMB groups (9 men, 16 women). The mean age at identification of first isolate in the multiple NTMB group was 62.2 years (men: 62.5 years (range 26-78); women: 62.1 years (range 43-85)). In contrast, the single NTMB group was younger (mean age 55.1 years; men: 50.5 years, women: 58.1 years), as were those with non-bronchiectasis lung disease and multiple isolates (mean age 47.2 years).

In the multiple NTMB group 52\% were non-smokers and $40 \%$ were ex-smokers. Only $8 \%$ of patients with bronchiectasis were current smokers compared with $27 \%$ of patients with non-bronchiectasis lung disease. $24 \%$ of the multiple NTMB group were on oral steroids at the time of the first isolate and $16 \%$ had a history of lobectomy. There was one patient of non-Caucasian origin in each group.

Sputum samples were sent at the time of first isolate for a variety of reasons: $32 \%$ were referred to our unit with known NTM, $28 \%$ were sent during routine surveillance, $16 \%$ due to suggestive CT changes, 16\% due to suggestive symptoms (cough, weight loss, fever, increased sputum, malaise, breathlessness), and only $8 \%$ because of failure to respond to usual bronchiectasis treatment.

\section{Comparison of NTM species in the three patient groups in the retrospective study}

MAC was by far the most common species identified in the multiple and single NTMB groups (table 1); in the nonbronchiectasis group $M$ kansasii and $M$ xenopi predominated. 
Table 1 Comparison of species isolated in the three groups in the retrospective study

\begin{tabular}{|c|c|c|c|}
\hline & $\begin{array}{l}\text { Bronchiectasis with } \\
\text { multiple NTM isolates } \\
(\mathrm{N}=25)\end{array}$ & $\begin{array}{l}\text { Bronchiectasis with } \\
\text { single NTM isolates } \\
(\mathrm{N}=23)\end{array}$ & $\begin{array}{l}\text { Non-bronchiectasis with } \\
\text { multiple NTM isolates } \\
(\mathrm{N}=22)\end{array}$ \\
\hline$M$ avium complex (MAC) & $18(72)$ & $10(43.5)$ & $2(9.0)$ \\
\hline M kansasii & $1(4)$ & $5(21.5)$ & $9(40.9)$ \\
\hline M chelonae & $2(8)$ & $4(17.4)$ & 0 \\
\hline M fortuitum & $2(8)$ & $1(4.4)$ & $1(4.5)$ \\
\hline $\mathrm{M}$ malmoense & $2(8)$ & $2(8.7)$ & $3(13.6)$ \\
\hline M xenopi & 0 & $1(4.4)$ & $7(31.8)$ \\
\hline
\end{tabular}

The causes of bronchiectasis in the multiple NTMB group were $24 \%$ idiopathic, $24 \%$ post-tuberculous (all MAC), $12 \%$ rheumatoid arthritis (all MAC), and 8\% each for post childhood infections, post adult infections, and primary ciliary dyskinesia. There was one each of pulmonary lymphangioleiomyomatosis, pulmonary sarcoidosis, allergic bronchopulmonary aspergillosis, and a compound heterozygote for cystic fibrosis with normal sweat test ( $M$ chelonae). Patients were not tested for HIV, but none were in high risk groups for this condition nor had lymphopenia.

In six patients $(24 \%)$, all who cultured MAC, the bronchiectasis was considered to be a consequence of primary NTM infection. In these cases the possibility of pre-existing bronchiectasis before NTM infection could not be excluded. However, the NTM infection was judged to have either led to marked progression of disease on the HRCT scan or to have been the sole cause of the bronchiectasis. Three of these patients also had rheumatoid arthritis, and one was a middle aged woman with a persistent dry cough whose bronchiectasis was present in the middle lobes, a pattern which has been described as Lady Windermere syndrome. ${ }^{15}$

\section{Microbiological results of patients in the retrospective study}

Patients in the single NTMB group were more likely to be smear negative $(83.3 \%)$ than those in the multiple NTMB group $(15.7 \%, p<0.0001)$. In the multiple NTMB group, $84 \%$ of smear positive sputum samples grew MAC in first isolates compared with $33.3 \%$ of smear negative samples. All of the three plus smear positive sputum samples grew MAC; nonMAC species were much less likely to be smear positive $(15.7 \%)$.

Pseudomonas aeruginosa was a co-pathogen at the time of first isolation of NTM in 52\% of the multiple NTMB group, but isolation of $P$ aeruginosa in these patients was chronic in only $16 \%$. $61 \%$ of patients with multiple MAC isolates cultured $P$ aeruginosa compared with $28 \%$ of patients with non-MAC isolates. $S$ aureus was co-cultured frequently in the multiple NTMB group (7/25, 28\%). Co-culture of $P$ aeruginosa and $S$ aureus occurred less often in the single NTMB group (39\% and $21.7 \%$, respectively) than in the multiple NTMB group. Rarer co-pathogens in the multiple NTMB group included Haemophilus influenzae (12\%), Aspergillus fumigatus (4\%), Candida albicans (8\%), and Stenotrophomonas maltophilia $(4 \%)$.

\section{HRCT scan at time of first isolate in the retrospective study}

Twenty four of the 25 patients in the multiple NTMB group underwent HRCT scanning (one patient had severe bronchiectasis previously shown by bronchography). Twenty HRCT scans were performed within 6 months of identification of the first NTM isolate (17 of these were performed within 1 month of the first isolate). Two HRCT scans were performed 9 months before identification of the first NTM isolate, one 7 months after, and one 11 months after.

Most of the patients had widespread bronchiectasis (table 2). Because of numbers of patients, comparisons were made between MAC and non-MAC species. Nodules, mucus plugging, and dyshomogeneity were common features and a quarter had cavities. MAC accounted for five of the six patients with cavities and consolidation (83.3\%) and for $78.6 \%$ of nodules and mucus plugging compared with nonMAC species. Dyshomogeneity had a similar prevalence in both groups. Patients requiring treatment had more cavities $(33.3 \% \vee 9.1 \%)$, consolidation $(41.6 \% \vee 9.1 \%)$, and had more severe $(58.3 \% \vee 18.2 \%)$ and widespread (75\% $v$ 45.4\%) bronchiectasis than those who did not.

Table 2 HRCT appearances in patients with bronchiectasis and multiple NTM isolates in the retrospective study

\begin{tabular}{llll}
\hline & $\begin{array}{l}\text { All isolates } \\
(\mathbf{N}=24)\end{array}$ & $\begin{array}{l}\text { M avium complex } \\
(\mathbf{N}=17)\end{array}$ & $\begin{array}{l}\text { Non-M avium complex } \\
(\mathbf{N}=\mathbf{7})\end{array}$ \\
\hline $\begin{array}{lll}\text { Bronchiectasis distribution } \\
\text { Widespread }\end{array}$ & \\
Lower and middle lobes & $15(62.5)$ & \\
Lower lobes & $4(16.6)$ & & \\
Upper and middle lobes & $3(12.5)$ & & \\
Lower and upper lobes & $2(8.3)$ & \\
Localised & $2(8.3)$ & \\
Middle lobe and lingula & $3(12.5)$ & & \\
Upper lobe & $2(8.3)$ & & \\
Specific HRCT features & $1(4.2)$ & & \\
Nodules and mucus plugging & & & \\
Dyshomogeneity & $14(58.3)$ & 11 & 1 \\
Cavities & $11(45.8)$ & 6 & \\
Consolidation & $6(25)$ & 5 & \\
Infiltrates & $6(25)$ & 5 & \\
\hline Data are shown as number of patients with percentage of total in parentheses. & \\
\hline
\end{tabular}



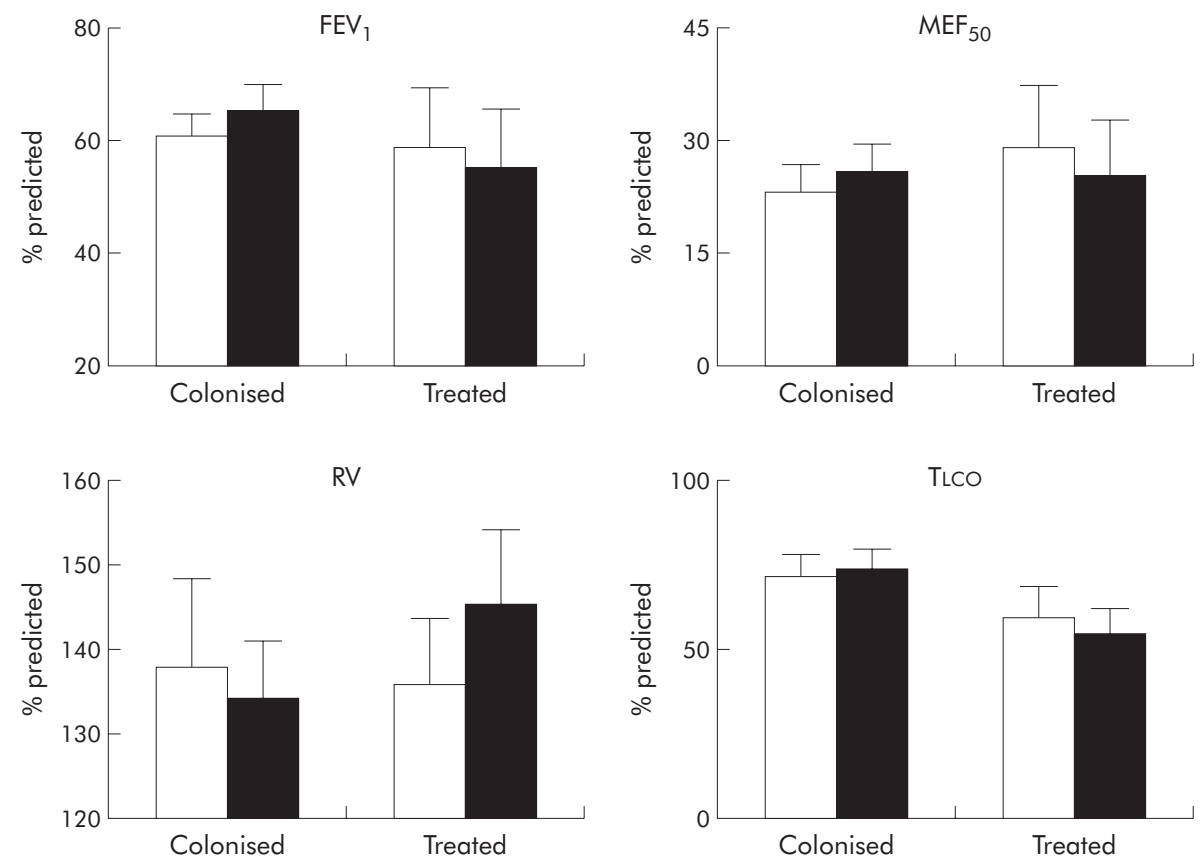

Figure 1 Mean (SE) lung function data (percentage predicted) in patients with bronchiectasis and multiple non-tuberculous mycobacteria isolates (NTMB). Lung function measured near the time of the first NTM isolate (open bars) was compared with the latest available lung function data (solid bars) for two groups of patients: a colonised group (mean 22.2 months after NTM isolation) and a drug treated group (mean 34.9 months after NTM isolation).

\section{Lung function in the retrospective study}

Lung function data were analysed for 22 patients in the multiple NTMB group (two patients had incomplete lung function data, one patient underwent surgery for NTM and was excluded). These were analysed as follows: those considered not to require treatment $(\mathrm{N}=11)$, those who had completed antimycobacterial treatment $(\mathrm{N}=5)$, and those continuing on antimycobacterial treatment $(\mathrm{N}=6)$ For each patient group, lung function data nearest to the time of the first NTM isolate was taken (median time -2 days before NTM isolate, range -8 to +11 months) and compared with the latest lung function data available.

In colonised patients the mean (SE) $\mathrm{FEV}_{1}$ was 60.7 (4.1)\% compared with $55.0(8.9) \%$ in drug treated patients at first isolate (difference non-significant; fig 1). Untreated patients with NTM had stable lung function, although there was a trend for increasing $\mathrm{FEV}_{1}$ and $\mathrm{MEF}_{50}$ and decreasing RV over time (difference non-significant). This might be due to general measures such as physiotherapy taken to improve the control of the bronchiectasis. Patients who had completed antimycobacterial treatment also had stable lung function (fig 2), while those still receiving antimycobacterial treatment showed a rise in RV over time (133\% rising to $150 \%$ ) and a fall in $\mathrm{MEF}_{50}(\mathrm{p}<0.05$; fig 3$)$.

\section{Management in the retrospective study}

Of the 25 patients in the retrospective study, 11 were considered not to require antibiotic treatment. Reasons for deciding to monitor rather than to treat included having a stable clinical condition with routine management and no HRCT evidence of active NTM disease. There was no species difference between the monitored and the treated group (64.3\% MAC in treated group $v 72.7 \%$ in the monitored group).

Of the remaining 14 patients, one underwent surgical resection of a cavity having previously completed 1 year of antimycobacterial treatment at the referring hospital and 13 were prescribed antimycobacterial drugs. The reasons for initiating treatment were multifactorial but HRCT changes influenced the decision in 11 of $13(84.6 \%)$. These were progressive bronchiectasis (36.4\%), new nodules $(27.3 \%)$, new/progression of cavities (36.4\%), and consolidation $(18.1 \%)$. Failure of symptoms to respond to usual treatment was a reason in $27.3 \%$; common symptoms in this regard were cough $(45.4 \%)$ and weight loss $(36.4 \%)$.

\section{DISCUSSION}

Patients are admitted to our minimal dependency unit for planned investigations ${ }^{2}$ at a time when their condition is stable, but they have usually been referred to our unit which is in a tertiary centre because their condition is causing concern. Similarly, patients followed up regularly in our clinics are usually more complicated than those seen in a general respiratory clinic.

The prevalence of NTM in our bronchiectatic population was $2 \%$. Previous studies have found a similar low prevalence, so it is likely that the prevalence of NTM in an unselected bronchiectasis population would be even lower than in our study. ${ }^{8-10}$

This is the first study to show that MAC is the predominant NTM species isolated from the sputum of patients with noncystic fibrosis bronchiectasis, both in single (45.5\%) and multiple isolates $(72 \%)$. This suggests that patients with bronchiectasis are susceptible to contracting MAC and, in some of these cases, long term colonisation or infection is established. In contrast, in our series and those of others, ${ }^{16}$ the species profile of NTM in patients without bronchiectasis is very different, with $M$ kansasii and $M$ xenopi predominating and MAC present in less than $10 \%$ of cases. ${ }^{16}{ }^{17} \mathrm{M}$ xenopi was found in only one single isolate and in no multiple isolates in patients with bronchiectasis. Single isolates are usually thought to represent environmental exposure just before the sample is taken. However, the predominance of MAC in single as well as multiple isolates suggests that, in 

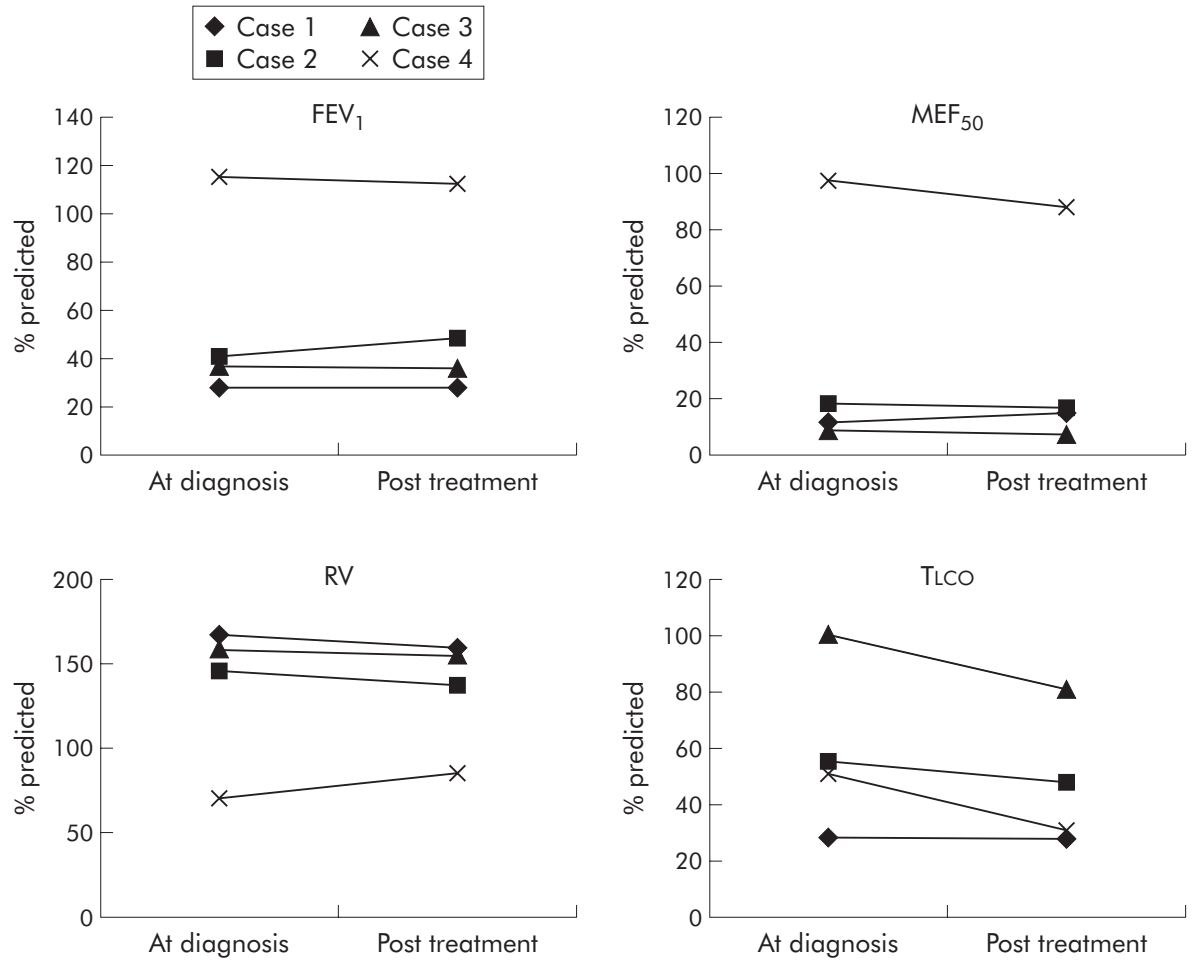

Figure 2 Mean (SE) lung function data (percentage predicted) in patients with bronchiectasis and multiple non-tuberculous mycobacteria isolates (NTMB) who had finished treatment and were sputum negative for NTM $(\mathrm{N}=4)$ after treatment. Each patient is represented as cases 1-4). Mean duration of treatment 16.7 months. Lung function measured near the time of first NTM isolate (diagnosis) was compared with latest available lung function data (mean 60 months later). Mean (SE) percentage predicted values at diagnosis and later are as follows: FEV, 54 (20) v56 (18); MEF $50: 33$ (21) v 31 (18); RV: 135 (21) v 135 (17); TLCO: 58 (15) v 47 (12).

- Case $1 \quad$ Case 3

- Case $2 \times$ Case 4
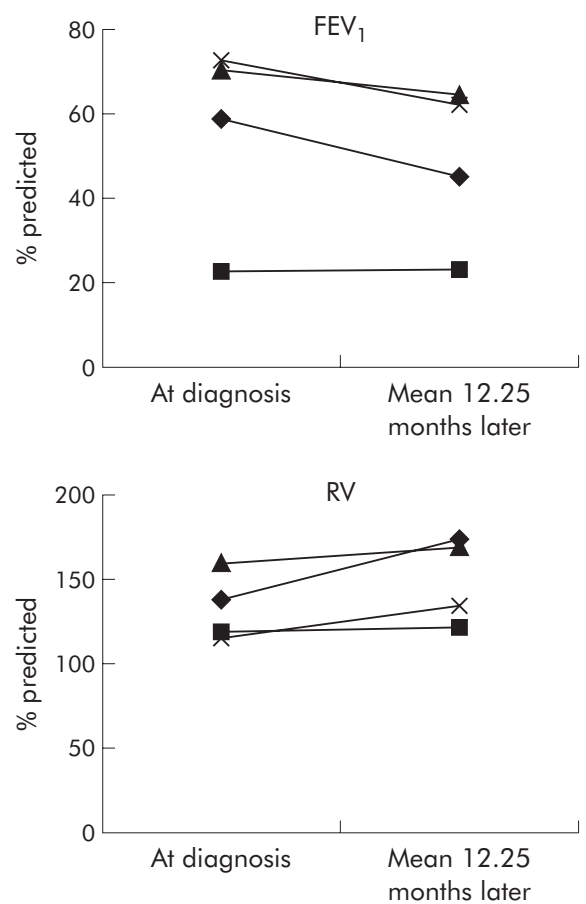
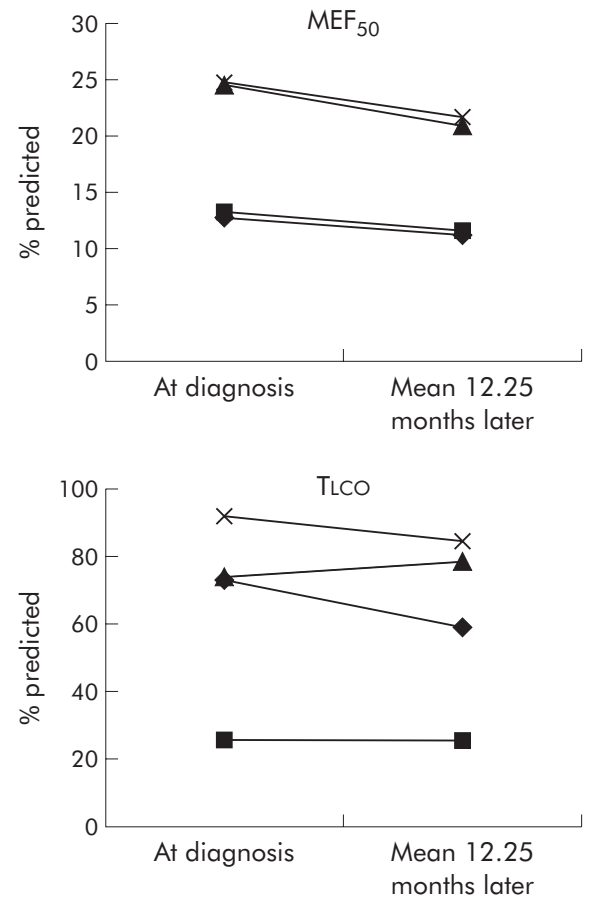

Figure 3 Mean (SE) lung function data (percentage predicted) in patients with bronchiectasis and multiple non-tuberculous mycobacteria isolates (NTMB) who were still on treatment and were sputum positive for NTM despite treatment (mean time on treatment 13.7 months; $N=4$ ). Each patient is represented as cases 1-4. Lung function measured near the time of the first NTM isolate (diagnosis) was compared with latest available lung function (mean 12.25 months later). Mean (SE) percentage predicted values at diagnosis and later are as follows: $\mathrm{FEV}_{1}: 56$ (11) v 49 (9); MEF $50: 18$ (3) v 16 (3), p<0.05; RV: 133 (10) v 150 (12); TLCO: 66 (14) v 62 (13). 
bronchiectasis, single isolates might represent temporary colonisation and the difference in species profile compared with non-bronchiectasis is probably due to unidentified factors causing a predisposition to infection. Since no particular cause of bronchiectasis was associated with NTM or MAC infection, this is likely to be due to the local environment of the bronchiectatic airway.

Pulmonary MAC disease is reported to present in two ways. $^{18}{ }^{19}$ The first is a classical presentation of fibrocavitatory lung disease in patients with either smoking related chronic obstructive pulmonary disease (COPD), previous tuberculosis, bronchiectasis, or cystic fibrosis. Those with smoking related COPD are predominantly men aged 50-70 years. ${ }^{1}$ A second form of MAC infection has been increasingly recognised to occur in apparently immunocompetent individuals with no pre-existing lung disease. ${ }^{18}{ }^{19}$ This group consists mainly of non-smoking women aged 40-80 years. These patients have no known pre-existing lung disease but HRCT scanning shows the presence of small nodules most commonly involving the middle lobe and mild cylindrical bronchiectasis. ${ }^{320}$ This presentation has been described as the Lady Windermere syndrome because habitual voluntary cough suppression is postulated to account for retained secretions and focal disease in the lingula and middle lobe. ${ }^{15}{ }^{21}$ After careful examination of the evidence available, we considered six of the 25 multiple NTMB cases to be primary infections. This was based on HRCT appearances, ${ }^{19} 2022$ the fact that no other cause of bronchiectasis was identified, ${ }^{2}$ and patient history including lack of chronic rhinosinusitis and profound tiredness which is present in most cases of idiopathic bronchiectasis. All six were primary infections with MAC. In primary infections it is usually impossible to be certain whether silent bronchiectasis predisposes to the MAC infection or whether the primary MAC infection itself causes the bronchiectasis in individuals who may have a genetic predisposition. ${ }^{22}$ Abnormalities in the gamma interferon pathway have recently been identified. ${ }^{23}$ HRCT studies of patients with MAC infection have shown small peripheral nodules which may coalesce and ectatic changes which develop in draining bronchi. ${ }^{14}$ Destruction of bronchial wall structure due to extensive granuloma formation in MAC lung infection has also recently been demonstrated. ${ }^{24}$

In our study the patients with bronchiectasis and multiple NTM isolates were predominantly female, which is also true of bronchiectasis in general. ${ }^{2}$ However, other features of the group were different from those reported in other bronchiectasis series. The mean age of the group (62.2 years) is higher than in other studies of bronchiectasis-for example, 50.4 years, ${ }^{8} 52.7$ years, ${ }^{10}$ and 41.6 years. ${ }^{25}$ The single NTMB group in our paper were younger, which suggests that patients with bronchiectasis may be less able to clear NTM to which they are exposed as they get older. Previous studies of bronchiectasis have suggested a negative smoking history with only $1 \%$ current smokers. ${ }^{25}$ The multiple NTMB group contained $40 \%$ ex-smokers and $8 \%$ current smokers. This association with cigarette smoking has also been noted with primary MAC infections in which $38 \%$ were ex-smokers. ${ }^{18}$ Treatment with regular oral corticosteroids and a history of previous lobectomy was also more common than is usual in a general bronchiectasis population. ${ }^{25}$

No particular aetiology of bronchiectasis was associated with NTM isolation. The prevalence of tuberculosis as a cause of bronchiectasis has been found to be $19 \%$ or less, ${ }^{8} 26$ and as low as $2 \% .{ }^{10}$ We found tuberculosis related bronchiectasis in patients with multiple NTM isolates to be more common $(24 \%)$, which suggests a possible increased general susceptibility to mycobacterial infection. ${ }^{27}{ }^{28}$ Similarly, the frequency of rheumatoid arthritis was higher than in other studies, ${ }^{10}$ either suggesting an immune mechanism for increased susceptibility to NTM or that rheumatoid associated small airways disease predisposes to NTM colonisation. ${ }^{29}$

Symptoms of cough, sputum production, and breathlessness are common in bronchiectasis so a high index of clinical suspicion is required to identify NTM. A large number of NTM isolates were detected by routine surveillance $(28 \%)$ and it is now our practice to screen our patients routinely once a year. Radiological appearances suggestive of NTM also accounted for a high proportion of NTM isolates, reflecting increased knowledge of the HRCT features of NTM lung disease. ${ }^{20}$

Pseudomonas aeruginosa was a frequent co-pathogen with MAC in patients with bronchiectasis and multiple NTM isolates $(52 \%)$ compared with other studies of bronchiectasis that have reported positive cultures in $20 \%, 26 \%$, and $31 \%$ of patients.[9. 10, 30] However, only a quarter of these were persistent isolates. Patients with bronchiectasis and single isolates also frequently co-cultured $P$ aeruginosa (39\%) with MAC. $P$ aeruginosa infection is associated with a greater extent of disease and worse lung function and may be a marker of patients whose lung function is declining. ${ }^{131}$ Co-culture of $P$ aeruginosa and MAC may therefore simply reflect worse bronchiectasis and resulting increased susceptibility to NTM. $S$ aureus is an uncommon pathogen in bronchiectasis and is associated with allergic bronchopulmonary aspergillosis and atypical variants of cystic fibrosis, ${ }^{32}$ both diseases which usually manifest as upper lobe disease. However, $S$ aureus was more frequently isolated in patients with bronchiectasis and NTM $(28 \%)$ than in other published studies. ${ }^{90} 30$ This suggests that repeated isolation of $S$ aureus in bronchiectasis should prompt a search for NTM as well as testing for allergic bronchopulmonary aspergillosis and atypical cystic fibrosis.

In a comparative study of CT appearances of patients infected with NTM species, higher numbers of nodules and more severe bronchiectasis were seen with MAC than with other NTM species. ${ }^{20}$ No differences were found in frequency of cavitation, consolidation, or "tree in bud" pattern between MAC and non-MAC species. Although the frequency of cavitation in our study was similar to that in a mixed lung disease population, ${ }^{20}$ we found that MAC accounted for the majority of cavities in bronchiectasis. This suggests that, in bronchiectasis, MAC behaves in a more aggressive fashion than non-MAC species, causing greater destruction of lung parenchyma and cavity formation.

Only just over half of the patients with bronchiectasis and multiple isolates were judged to require treatment. ${ }^{57}$ The rest were considered to be colonised but careful monitoring was continued, and our results show that lung function and radiology remained stable. Patients who required treatment had more severe impairment of lung function, and this got worse if patients remained on treatment usually due to failure of eradication. A key factor in the decision to treat was evidence of changes on HRCT scanning, especially progression of bronchiectasis and appearance of new cavities. A higher proportion of patients who went on to have treatment had cavities $(33.3 \% \vee 9.1 \%)$, consolidation $(58.3 \% \vee 9.1 \%)$, or had more widespread and severe bronchiectasis (75\% $v$ $45.4 \%)$.

In summary, we have shown that, although NTM are uncommon in bronchiectasis, infection can lead to worsening of the condition. A significant number of cases were detected by routine surveillance. MAC is the predominant species whatever the underlying aetiology, and is responsible for most of the cavitatory disease. Patients with bronchiectasis may be more susceptible to MAC. However, pulmonary MAC infection might progress early bronchiectasis into more severe disease in genetically susceptible individuals such as those with rheumatoid arthritis. A proportion of patients 
presenting with symptoms of bronchiectasis are probably caused by primary MAC infections in previously normal lungs. HRCT scanning is very important in aiding the decision about when to start treatment.

\section{Authors' affiliations}

M Wickremasinghe, L J Ozerovitch, G Davies, T Wodehouse, M V Chadwick, S Abdallah, P Shah, R Wilson, Host Defence Unit, Royal Brompton Hospital, London, UK

Competing interests: none declared.

\section{REFERENCES}

1 Aksamit TR. Mycobacterium avium complex pulmonary disease in patients with pre-existing lung disease. Clin Chest Med 2002;23:643-53.

2 Wilson R. Bronchiectasis. In: Gibson G, Geddes D, Costabal U, et al. Respiratory medicine. 3rd ed. London: W B Saunders, 2003: 1445-64.

3 Levin DL. Radiology of pulmonary Mycobacterium avium-intracellulare complex. Clin Chest Med 2002;23:603-12.

4 Catanzaro A. Diagnosis, differentiating colonization, infection, and disease. Clin Chest Med 2002;23:599-601

5 British Thoracic Society. Management of opportunist mycobacterial infections: joint tuberculosis committee guidelines 1999. Thorax 2000;55:210-8.

6 American Thoracic Society. Diagnosis and treatment of disease caused by nontuberculous mycobacteria. Am J Respir Crit Care Med 1997; 156:S1-25.

7 British Thoracic Society. First randomised trial of treatments for pulmonary disease caused by $M$ avium intracellulare, $M$ malmoense, and $M$ xenopi in HIV negative patients: rifampicin, ethambutol and isoniazid versus rifampicin and ethambutol. Thorax 2001;56:167-72.

8 Chan CH, Ho AK, Chan R, et al. Mycobacteria as a cause of infective exacerbation in bronchiectasis. Postgrad Med J 1992;68:896-9.

9 Palwatwichai A, Chaoprasong C, Vattanathum A, et al. Clinical, laboratory findings and microbiologic characterization of bronchiectasis in Thai patients. Respirology 2002;7:63-6.

10 Pasteur MC, Helliwell SM, Houghton SJ, et al. An investigation into causative factors in patients with bronchiectasis. Am J Respir Crit Care Med 2000;162:1277-84.

11 Collins CH, Lyne PM, Grange JM, et al. Microbiological methods. 8th ed. London: Arnold, 2004

12 American Society of Microbiology. Manual of clinical microbiology, 7th ed American Society of Microbiology, 1999.

13 Roberts HR, Wells AU, Milne DG, et al. Airflow obstruction in bronchiectasis: correlation between computed tomography features and pulmonary function tests. Thorax 2000;55:198-204.
14 Tanaka E, Amitani R, Niimi A, et al. Yield of computed tomography and bronchoscopy for the diagnosis of Mycobacterium avium complex pulmonary disease. Am J Respir Crit Care Med 1997; 155:2041-6.

15 Reich JM, Johnson RE. Mycobacterium avium complex pulmonary disease presenting as an isolated lingular or middle lobe pattern. The Lady Windermere syndrome. Chest 1992;101:1605-9.

16 Griffith DE. Management of disease due to Mycobacterium kansasii. Clin Chest Med 2002;23:613-21.

17 Olivier KN. Nontuberculous mycobacterial pulmonary disease. Curr Opin Pulm Med 1998;4:148-53

18 Prince DS, Peterson DD, Steiner RM, et al. Infection with Mycobacterium avium complex in patients without predisposing conditions. N Engl J Med 1989;321:863-8.

19 Field SK, Fisher D, Cowie RL. Mycobacterium avium complex pulmonary disease in patients without HIV infection. Chest 2004;126:566-81.

20 Hollings NP, Wells AU, Wilson R, et al. Comparative appearances of non-tuberculous mycobacteria species: a CT Study. Eur Radiol 2002;12:2211-7.

21 Reich JM, Johnson RE. Mycobacterium avium complex lung disease in women. Chest 1995; 107:293-5.

22 McKlendin K, Stark P. Mycobacterium avium intracellulare complex as a cause of bronchiectasis. Semin Respir Infect 2001;16:85-7.

23 Safdar A, Armstrong D, Murray HW. A novel defect in interferon-gamma secretion in patients with refractory nontuberculous pulmonary mycobacteriosis. Ann Intern Med 2003;138:521.

24 Fujita J, Ohtsuki Y, Shigeto E, et al. Pathological findings of bronchiectasis caused by Mycobacterium avium intracellulare complex. Respir Med 2003;97:933-8.

25 Cole P. Bronchiectasis. In: Brewis RAL, Corrin B, Geddes DM, et al, eds. Respiratory medicine. 2nd ed. London: W B Saunders, 1995:1286-316.

26 Barker AF, Bardana EJ Jr. Bronchiectasis: update of an orphan disease. Am Rev Respir Dis 1988;137:969-78.

27 Roach TI, Chatteriee D, Blackwell JM. Induction of early-response genes KC and JE by mycobacterial lipoarabinomannans: regulation of $\mathrm{KC}$ expression in murine macrophages by Lsh/lty/Bcg (Candidate Nramp). Infect Immun 1994;62:1176-84.

28 Barker AF. Bronchiectasis. N Engl J Med 2002;346:1383-93.

29 Perez T, Remy-Jardin M, Cortet B. Airways involvement in rheumatoid arthritis: clinical, functional, and HRCT findings. Am J Respir Crit Care Med 1998; 157:1658-65

30 Angrill J, Agusti C, de Celis R, et al. Bacterial colonisation in patients with bronchiectasis: microbiological pattern and risk factors. Thorax 2002;57:15-9.

31 Evans SA, Turner SM, Bosch BJ, et al. Lung function in bronchiectasis: the influence of Pseudomonas aeruginosa. Eur Respir J 1996;9:1601-4.

32 Shah PL, Mawdsley S, Nash K, et al. Determinants of chronic infection with Staphylococcus aureus in patients with bronchiectasis. Eur Respir J $1999 ; 14: 1340-4$ 